LAPP-EXP 2002-07

December 2002

\title{
LEP constraints on SUSY Dark Matter
}

\author{
S. Rosier-Lees \\ LAPP, IN2P3-CNRS, Chemin de Bellevue, BP110, \\ F-74941, Annecy-le-Vieux
}

\begin{abstract}
A wide range of supersymmetric searches has been performed at LEP. The negative outcome of those searches has been translated into indirect constraints on the lightest neutralino, $\chi_{1}^{0}$, a good supersymmetric candidate for the cold dark matter. After presenting the LEP experimental environment, the $\chi_{1}^{0}$ lower mass limit within different theoretical assumptions will be reviewed.
\end{abstract}

Talk given at the $4^{\text {th }}$ International Workshop of the Identification of Dark Matter, IDM2002, 2-4 September 2002, York, UK 


\title{
LEP CONSTRAINTS ON SUSY DARK MATTER
}

\author{
SYLVIE ROSIER-LEES \\ LAPP, Annecy-Le-Vieux, IN2P3-CNRS, France \\ e-mail: rosier@lapp.in2p3.fr
}

\begin{abstract}
A wide range of supersymmetric searches has been performed at LEP. The negative outcome of those searches has been translated into indirect constraints on the lightest neutralino, $\tilde{\chi}_{1}^{0}$, a good supersymmetric candidate for the cold dark matter. After presenting the LEP experimental environment, the $\tilde{\chi}_{1}^{0}$ lower mass limit within different theoretical assumptions will be reviewed
\end{abstract}

\section{Introduction}

Supersymmetry theory provides a good candidate to the cold dark matter: the Lightest Supersymmetric Particle (LSP). Under the R-parity conservation assumption the LSP is stable. Due to the strong experimental constraints on stable charged particles, the LSP is most probably neutral. The ligthest neutralino $\tilde{\chi}_{1}^{0}$ is a good LSP candidate and gives relic density $\Omega h^{2}$ compatible with measurements for a wide range of the parameter space. Since the $\mathrm{Z} \tilde{\chi}_{1}^{0} \tilde{\chi}_{1}^{0}$ coupling is suppressed and the $\tilde{\chi}_{1}^{0}$ escapes detection (neutral and weakly interacting), the $\tilde{\chi}_{1}^{0} \tilde{\chi}_{1}^{0}$ direct search at LEP was disfavoured. Nevertheless, the LEP sensitivity for chargino, slepton or stop and sbottom quark searches was optimal. By exploiting te mass and coupling of each SUSY particle dependence with the SUSY parameters, indirect constraints on the $\tilde{\chi}_{1}^{0}$ mass have been derived. The robustness of those constraints is discussed for the different theoretical model assumptions.

\section{Searches at LEP}

\subsection{The Large Electron Positron collider: LEP}

The LEP accelerator the CERN synchroton electron-positron collider, has started operation in 1989. The data taking has ended on November 2000. The two phases of LEP operation were:

- LEP1 (1989-1995) where millions of Z events have been accumu- 
lated for high precision measurements with an integrated luminosity of roughly 175 inverse picobarn $\left(\mathrm{pb}^{-1}\right)$ collected per experiment.

- LEP2 (1996-2000) where the centre-of-mass energy $(\sqrt{s})$ has been increased from $130 \mathrm{GeV}$ to $208 \mathrm{GeV}$ extending the new particle search area. In the meantime the integrated luminosity collected per year and per experiment increased from $5 \mathrm{pb}^{-1}$ to $250 \mathrm{pb}^{-1}$. LEPII was a real success and want far beyond the nominal goals.

\subsection{The Four LEP experiments: $A D L O$}

Four experiments have been installed on the LEP: ALEPH, DELPHI, L3 and OPAL. Each detector was designed to cover a large solid angle, to identify and reconstruct electrons, photons, muons, to tag heavy flavor $(\tau$ and b-quark) and to reconstruct hadron jet. In contrast with the hadron colliders, the trigger criteria were simple and all the physics was recorded leading to a trigger efficiency of $100 \%$ (for events with a deposited energy greater than $3 \mathrm{GeV}$ ).

\section{Sensitivities}

The sensitivity is a function of the signal cross section production, the signal efficiency and the background. The visible energy and the particle multiplicity will be almost proportional to the mass difference between the SUSY particle and the $\tilde{\chi}_{1}^{0}$ :

$$
\Delta M=M_{\tilde{\mathrm{P}}}-M_{\tilde{\chi}_{1}^{0}}
$$

Thus the search strategy and the sensitivity will depend on this $\Delta M$ parameter.

\subsection{Cross section production}

- Chargino and Neutralino: Charginos $\left(\tilde{\chi}_{1,2}^{ \pm}\right)$, the supersymmetric partners of $\mathrm{W}^{ \pm}$and $\mathrm{H}^{ \pm}$, are pair produced via $s$-channel $\gamma / \mathrm{Z}$ exchange. The production cross section can be reduced by an order of magnitude when the $t$-channel scalar neutrino $(\tilde{\nu})$ exchange is important. Neutralinos, the supersymmetric partners of $\mathrm{Z}, \gamma$, and neutral Higgs bosons, are pair produced $\mathrm{e}^{+} \mathrm{e}^{-} \rightarrow \tilde{\chi}_{i}^{0} \tilde{\chi}_{j}^{0}(i, j=$ $1, \ldots, 4$; ordered by their masses) via $s$-channel $\mathrm{Z}$ exchange and their production cross section can be enhanced by $t$-channel exchange of a scalar electron $\left(\tilde{\mathrm{e}}^{ \pm}\right)$. 
- Slepton pairs: Scalar leptons $\left(\tilde{\ell}_{R}^{ \pm}\right.$and $\left.\tilde{\ell}_{L}^{ \pm}\right)$are the supersymmetric partners of the right- and left-handed leptons. Pair production takes place through $s$-channel $\gamma / \mathrm{Z}$ exchange. For scalar electrons the production cross section is enhanced by $t$-channel exchange of a neutralino.

\subsection{SUSY event topologies}

The list of the processes accessible at LEPII is given in Table 1 as well as the decay channels. The mean features of the SUSY searches performed at LEP can be summarized as follows:

- Under R-parity conservation each particle are pair produced and decays into the $\tilde{\chi}_{1}^{0}$ and their associated partner of the Standard Model. Since the $\tilde{\chi}_{1}^{0}$ escapes detection, the SUSY final state topologies are characterized by missing energy and acoplanar objects (hadronic jets or leptons). The key point for each LEP detector was the hermiticity.

- The number of final states is large: ranging from the simple acoplanar lepton pair to the multijets plus missing energy final state or photon plus missing energy events. This large variety of explored signatures was the strength of the SUSY searches performed at LEP. Thus the complementarity of the SUSY processes was exploited in an optimal way.

\subsection{Background}

The signal topologies and the associated background sources change for different $\Delta M$ ranges. In the low and very low $\Delta M$ ranges, the expected topologies for the signal are characterized by a low multiplicity and a low visible energy, and the background is dominated by the two-photon interactions. For high $\Delta M$ ranges, the signal signatures are very similar to those of W-pair production; in particular for $\Delta M>80 \mathrm{GeV}$ on-shell Ws are produced. For intermediate $\Delta M$ the best sensitivity is achieved since the background is minimal. The cross section production measurements at different centre-of-mass energies for each process are in a very good agreement with theoretical predictions as depicted on Fig. 1 (left side). 


\begin{tabular}{|c|c|c|}
\hline Processus & Decay channel & Final State-Topology \\
\hline \multirow[t]{4}{*}{$\mathrm{e}^{+} \mathrm{e}^{-} \rightarrow \tilde{\chi}_{1}^{+} \tilde{\chi}_{1}^{-}$} & $\tilde{\chi}_{1}^{+} \rightarrow \mathrm{W}^{*+} \tilde{\chi}_{1}^{0}$ & \\
\hline & $\mathrm{W}^{*+} \rightarrow l^{+} \nu$ & Acoplanar lepton pairs plus $\mathscr{E}$ \\
\hline & $\mathrm{W}^{*+} \rightarrow q^{1} \bar{q}^{2}$ & Four hadronic jets plus $\not E$ \\
\hline & $\mathrm{W}^{*+} \rightarrow q^{1} \bar{q}^{2}$ & Two jets plus one lepton plus $\not \notin$ \\
\hline \multirow[t]{2}{*}{$\mathrm{e}^{+} \mathrm{e}^{-} \rightarrow \tilde{\chi}_{1}^{+} \tilde{\chi}_{1}^{-}\left(\gamma_{\mathrm{isr}}\right)$} & $\tilde{\chi}_{1}^{+} \rightarrow \mathrm{W}^{*+} \tilde{\chi}_{1}^{0}$ & $\gamma$ plus soft particle $+\not \notin$ \\
\hline & $M_{\tilde{\chi}_{1}^{+}} \sim M_{\tilde{\chi}_{1}^{0}}$ & \\
\hline \multirow[t]{3}{*}{$\mathrm{e}^{+} \mathrm{e}^{-} \rightarrow \tilde{\chi}_{j_{\geq 2}}^{0} \tilde{\chi}_{i_{\geq 1}}^{0}$} & $\tilde{\chi}_{j \geq 2}^{0} \rightarrow \mathrm{Z}^{*+} \tilde{\chi}_{1}^{0}$ & \\
\hline & $\mathrm{Z}^{*} \rightarrow l^{+} l^{-}$ & Acoplanar lepton pairs plus $\mathbb{E}$ \\
\hline & $\mathrm{Z}^{*} \rightarrow q \bar{q}$ & Acoplanar jet pairs plus $\not \subset$ \\
\hline $\mathrm{e}^{+} \mathrm{e}^{-} \rightarrow \tilde{\chi}_{2}^{0} \tilde{\chi}_{1}^{0}$ & $\tilde{\chi}_{2}^{0} \rightarrow \gamma+\tilde{\chi}_{1}^{0}$ & Photon plus $\not E$ \\
\hline \multirow[t]{2}{*}{$\mathrm{e}^{+} \mathrm{e}^{-} \rightarrow \tilde{\ell}^{+} \tilde{\ell}^{-}$} & $\tilde{\ell}^{+} \rightarrow l^{+} \tilde{\chi}_{1}^{0}$ & Acoplanar lepton pairs plus $\not{E}$ \\
\hline & $l=\mu, e, \tau$ & \\
\hline \multirow[t]{2}{*}{$\mathrm{e}^{+} \mathrm{e}^{-} \rightarrow \tilde{\mathrm{q}} \tilde{\overline{\mathrm{q}}}$} & $\tilde{\mathrm{q}} \rightarrow q+\tilde{\chi}_{1}^{0}$ & Acoplanar hadronic pairs plus $\not E$ \\
\hline & $\tilde{\mathrm{q}}=\tilde{\mathrm{t}_{1}}, \tilde{\mathrm{b}}$ & \\
\hline
\end{tabular}

\subsection{Results}

No excess of events has been observed in any channel and any experimen thus upper limits on the chargino, neutralino and slepton production cross sections have been set. Exclusion limits at 95\% C.L. are derived taking into account background contributions and derived in the $\left(M_{\tilde{\chi}_{1}^{0}, M_{\tilde{\mathrm{P}}}}\right)$ plane since the detection sensitivity depends on both parameters. The "standard" chargino search sensitivity is illustrated on Fig. 1: the best sensitivity is achieved for the intermediate $\Delta M$ regions, where the background is low and the efficiency greater than $40 \%$ leading to cross section upper limits as low as $0.15 p b^{-1}$. For large $\Delta M$ values, the signal efficiency is still high $(\sim 40 \%)$ but the WW background remains irreducible leading to cross section upper limits ranging from 0.5 to $0.7 p b^{-1}$. For the low $\Delta M$ values, it becomes even worst dealing with a huge background and a poor trigger efficiency: low particle multiplicity and low deposited energy make the trigger inefficient. The sensitivity becomes very low yielding cross section upper limits of the 
order of $2 p b^{-1}$ for $\Delta M \sim 5 \mathrm{GeV}$.

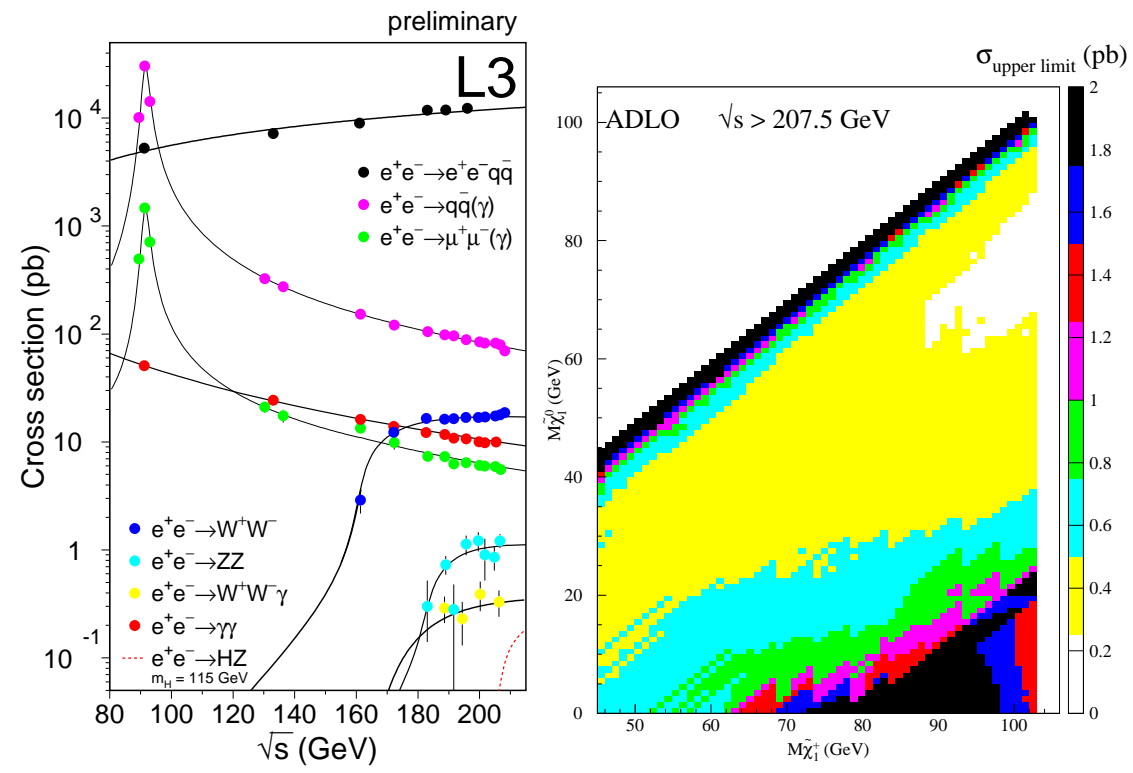

Figure 1. Left: Cross section production measurements at different centre-of-mass energies for each process of the Standard Model 3

Right: The Observed Upper limit on the $\mathrm{e}^{+} \mathrm{e}^{-} \rightarrow \tilde{\chi}_{1}^{+} \tilde{\chi}_{1}^{-}$cross section production assuming $\mathrm{a} \operatorname{br}\left(\tilde{\chi}_{1}^{1} \rightarrow \mathrm{W}^{*} \tilde{\chi}_{1}^{0}\right)=100 \%$ with $\mathrm{ADLO}$ at the highest centre-of-mass energy

In the $\Delta M$ regions below $3 \mathrm{GeV}$, classical SUSY searches, so called standard, were blind. Introducing the Initial State Radiation (ISR)- $\gamma$ analysis, some sensitivity has been recovered by increasing the trigger efficiency and reducing the two-photon interaction background (despite a $90 \%$ reduction of the signal cross section production). Upper limits on slepton cross section productions have been also derived and combined early by the four LEP experiments ${ }^{5}$ since for scalar particles the integrated luminosity matters above all.

\section{4. $\tilde{\chi}_{1}^{0}$ mass limits}

In the MSSM, with Grand Unification assumption, the masses and couplings of the SUSY particles as well as their production cross sections, are entirely described once five parameters are fixed: $\tan \beta$ (the ratio of the 
vacuum expectation values of the two Higgs doublets), $M \equiv M_{2}$ (the gaugino mass parameter), $\mu$ (the higgsino mixing parameter), $m_{0}$ (the common mass for scalar fermions at the GUT scale) and $A$ (the trilinear coupling in the Higgs sector). We investigate the following MSSM parameter space:

$$
\begin{aligned}
& 0.7 \leq \tan \beta \leq 60, \\
& 0 \leq M_{2} \leq 2000 \mathrm{GeV}, \\
& -2000 \mathrm{GeV} \leq \mu \leq 2000 \mathrm{GeV}, 0 \mathrm{GeV} \leq m_{0} \leq 500 \mathrm{GeV} .
\end{aligned}
$$

The impact of the maximal mixing in the stau sector has not been included in what follows but has been studied in ${ }^{1},{ }^{2}$ and ${ }^{3}$ experiments where it was shown that the limits remain robust.

To derive the absolute mass limits of the lightest neutralino, a scan in the MSSM parameter space is performed in steps of $0.2 \mathrm{GeV}$ for $M_{2}, 1.0$ $\mathrm{GeV}$ for $\mu$ and $0.5 \mathrm{GeV}$ for $m_{0}$.

All the limits on the cross sections have been translated into exclusion regions in the MSSM parameter space. To derive such limits, the cross sections and branching fractions have been calculated in the MSSM framework.

In what follows the $\mu$ parameter is free (No dynamic EWSB) which is equivalent to vary $A_{0}$ in fact. When imposing the dynamic EWSB, $\mu$ but the sign is no more a free parameter and we are in the more constrained mSUGRA framework. Exhaustive interpretations of the LEP results has been performed in this framework and are reviewed in ${ }^{6}$ but will not be described in what follows.

\subsection{Large scalar mass or Large $m_{0}$}

The slepton within this assumption are out of the kinematic reach, the mean sensitivity is given by the chargino searches. Since the cross section production depends on the chargino field contents, different $\tilde{\chi}_{1}^{0}$ mass limits are set.

- Deep Gaugino Chargino $\left(\mu>>M_{2}\right)$ : This is the optimal scenario, the cross section production is the highest, the $\Delta M$ intermediate leading to the best sensitivity: the chargino is at the kinematic reach thus it is important to reach the highest center of mass energy. Combining the four LEP experiments at $\sqrt{s}>207.5 \mathrm{GeV}$ with an overall luminosity of $32 \mathrm{pb}^{-1}$ chargino ligther than $103.5 \mathrm{GeV}$ is excluded leading to $\tilde{\chi}_{1}^{0}$ mass limit of $52 \mathrm{GeV}^{5}$.

- Deep Higgsino Chargino $\left(\mu>>M_{2}\right)$ : Within the scenario, the limit on chargino mass is obtained when the chargino and the $\tilde{\chi}_{1}^{0}$ 
are mass degenerate. A limit independent on $\Delta M$ on the chargino mass has been set by each experiment: $M_{\tilde{\chi}_{1}^{ \pm}}>85 \mathrm{GeV}$, thanks to the ISR $\gamma$ analysis. The four LEP experiments are going to combine their results, a final limit of the order $90-95 \mathrm{GeV}$ is expected. Thus the limit on the $\tilde{\chi}_{1}^{0}$ mass should be greater than $90 \mathrm{GeV}$ in this scheme.

- Mixed Higgsino-Gaugino Chargino $\left(\mu \sim M_{2}\right)$ : For a given chargino mass, the mass of the $\tilde{\chi}_{1}^{0}$ is minimal for $\mu \sim-M_{2}$ and $\tan \beta=1$. For those parameter ranges, the chargino and neutralino searches are complementary as depicted in Fig 2, left side, where the neutralino process allow to go beyond the chargino kinematic limit. $\tilde{\chi}_{1}^{0}$ mass limits ranging from 37 to $39.6 \mathrm{GeV}$ are obtained by the four experiments. The $\tan \beta$ dependence is illustrated on Fig. 2 on the right side.
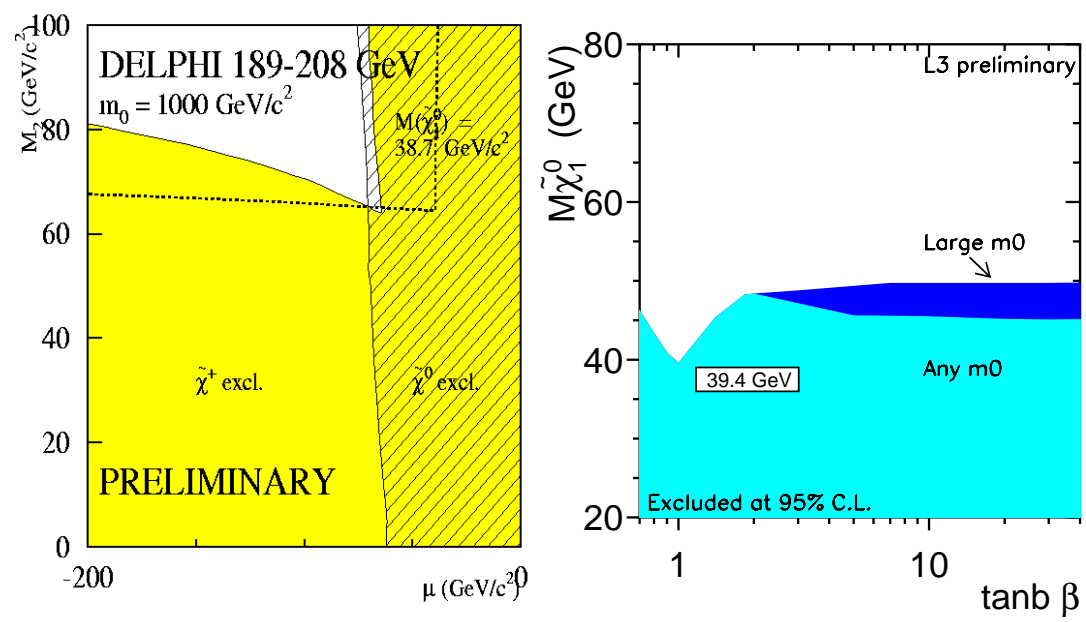

Figure 2. $\tilde{\chi}_{1}^{0}$ mass limit in the $\left(\mu, M_{2}\right)$ plane for $\tan \beta=1{ }^{2}$ (left side) and as a function of $\tan \beta{ }^{3}$ (right side)

\subsection{Low scalar mass or Low $m_{0}$}

For low $m_{0}$ values, sleptons are light and within reach of LEP energies. Due to the $\beta^{3}$ dependence of the cross section production, the collected 
luminosity matters mainly. For this reason, LEP analyses were combined at the very beginning of the LEP working group activity. The selectron, smuon and stau mass limits are respectively $99.4,96.4$ and $87.1 \mathrm{GeV}$ assuming a $\tilde{\chi}_{1}^{0}$ mass of $40 \mathrm{GeV}{ }^{5}$.

When combining slepton, chargino and neutralino searches, the weakest sensitivity is found to be when the $\tilde{\chi}_{1}^{ \pm}$, the $\tilde{\nu}$ and the $\tilde{\chi}_{1}^{0}$ are mass degenerate: $\tilde{\chi}_{1}^{+}$becomes invisible and is produced with a low rate, $\tilde{\chi}_{2}^{0}$ decaying into $\tilde{\nu}, \nu$ is also invisible, the sensitivity is then recovered with the sleptons. The indirect limit on the $\tilde{\chi}_{1}^{0}$ mass is then given through the $M_{2}$ dependence on the selectron mass. On Fig 2 left side, one can see how the $\tilde{\chi}_{1}^{0}$ mass limit is affected at high $\tan \beta$ values when scanning over all the $m_{0}$ values.

\subsection{The impact of Higgs boson search}

At the tree level, the lighter CP-even Higgs boson mass depends only on the mass $m_{A}$ of the CP-odd Higgs boson and on $\tan \beta$. The value of $m_{h}$ ranges from zero for $m_{A}=0$, to a maximum value of $m_{Z}|\cos 2 \beta|$ for very large values of $m_{A}$. Thus, a lower limit on $m_{h}$ can be translated into a lower limit on $\tan \beta$. When including the radiative corrections due to the large top quark mass, $m_{h}^{2}$ receives an additional contribution proportional to $m_{t}^{4} \log \left(m_{\tilde{\mathrm{t}}}^{2}\right)$, assuming the two stops are mass-degenerate. It appears that for stop not much heavier than top quark, this correction remains small to still allow a lower limit on $\tan \beta$ to be derived ${ }^{1}$. Alternatively, for low $\tan \beta$ value, a minimum value for $m_{\tilde{\mathrm{t}}}$ can be inferred from the lower limit on $m_{h}$. This $m_{\tilde{\mathrm{t}}}$ lower limit through its dependence with $M_{2}$ can be translated into a lower limit on the $\tilde{\chi}_{1}^{0}$ mass for a given range of $m_{0}$.

As can be seen in Fig 3, the $\tilde{\chi}_{1}^{0}$ mass limit is improved in the low $\tan \beta$ region with the Higgs boson searches leading to a mass limit of $50 \mathrm{GeV}$ for $\tan \beta$ values less than 3 . Finally the absolute lower mass limit on the $\tilde{\chi}_{1}^{0}$ is found to be equal to $47 \mathrm{GeV}$ resulting from the combination of the Slepton, Higgs boson and Chargino searches performed by the ADLO experiments ${ }^{1}$ and ${ }^{5}$. This limit holds for any $m_{0} \leq 1 \mathrm{TeV}$ and $m_{t}=175 \mathrm{GeV}$. For $m_{0}$ values larger than $2 \mathrm{TeV}$, the Higgs boson searches no longer improve on the $\tilde{\chi}_{1}^{0}$ mass limit at low $\tan \beta$ deduced from with chargino and neutralino searches.

\section{Conclusion}

A wide variety of searches has been carried out at LEP2 and despite all the efforts, no indication for new physics has been observed up to $208 \mathrm{GeV}$. 
LEP2 was an unique place for SUSY searches: Due to the good detector performances and the relatively good knowledge of the background reactions, a large domain has been covered and all channels have been exploited. With the absence of any signal, LEP2 excluded chargino masses up to the kinematic limit $(103.5 \mathrm{GeV})$ for most of the SUSY parameter space, even better an absolute limit has been obtained $\sim 90 \mathrm{GeV}$ irrespective of $\Delta M$ value. In addition, an indirect and absolute lower limit, equal to $39.6 \mathrm{GeV}$ on the Lightest Susy Particle has been derived in the framework of the CMSSM. By including the Higgs searches, this limit becomes $47 \mathrm{GeV}$ and is obtained at large $\tan \beta$ values but holds for any $m_{0} \leq 1 \mathrm{TeV}$ and $m_{t}=175$ $\mathrm{GeV}$. In order to test the robustness of this limit, a first attempt has been made to relax the gaugino mass unification assumption by decreasing the parameter $\mathrm{R} 12=M 1 / M 2$ from 0.55 (value at the EW scale within the GUT unification assumption) to 0.1 values. By combining the constraint on the relic density $\Omega h^{2}<0.3{ }^{7}$ with the LEP constraints the $\tilde{\chi}_{1}^{0}$ mass limit becomes $12 \mathrm{GeV}$ irrespective of R12 as depicted on Fig 3 .

\section{Acknowledgements}

It is a pleasure for me to thank the organizers of the IDM2002 conference for the very stimulating sessions and for the very pleasant atmosphere at the conference.
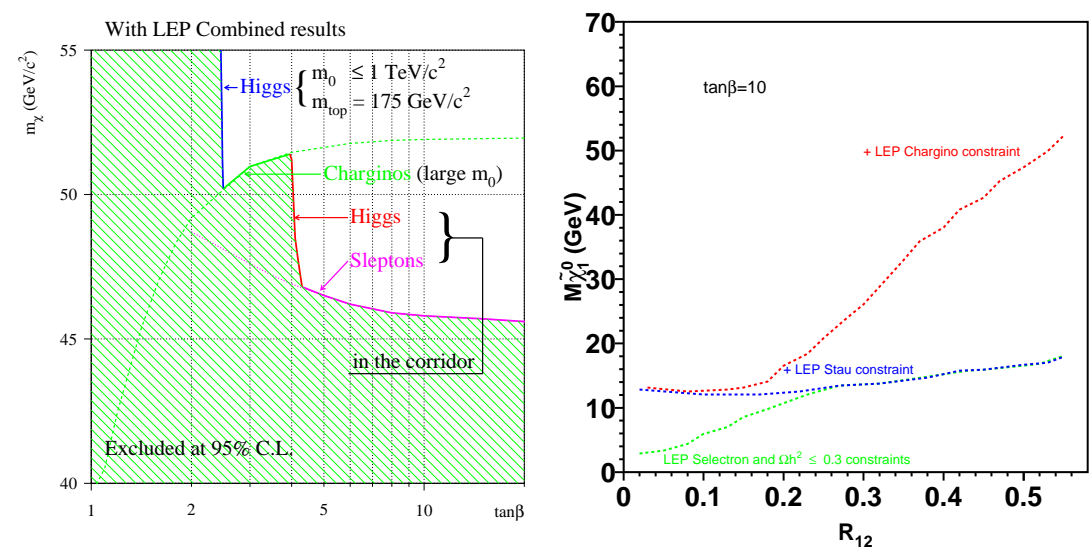

Figure 3. Mass limit on the $\tilde{\chi}_{1}^{0}$ including Chargino, Higgs and sleptons searches at LEP ${ }^{5}$ (left side) and when relaxing the gaugino mass unification at the GUT scale ${ }^{7}$ (right side) 


\section{References}

1. ALEPH experiment on the WEB under, http://Alpehwww.cern.ch/

2. DELPHI experiment on the WEB under, http://Delphiwww.cern.ch/

3. L3 experiment on the WEB under, http://13www.cern.ch/

4. OPAL experiment on the WEB under, http://opalwww.cern.ch/

5. The LEP SUSY working group on the WEB under, http://lepsusy.web.cern.ch/lepsusy

6. J.Ellis review on the constraints from the accelerators and relic density in the mSUGRA framework, these proceedings.

7. micrOMEGAS G.Belanger, F.Boudjema, A.Pukhov and A.Semenov, hep$\mathrm{ph} / 0112278$ 